\title{
ПОВЫШЕНИЕ ЭНЕРГЕТИЧЕСКОЙ И ЭКОЛОГИЧЕСКОЙ ЭФФЕКТИВНОСТИ СИСТЕМ ГАЗООЧИСТКИ НА ТЭС
}

\author{
Замалиева Альбина Таврисовна', \\ Albina-0587@rambler.ru \\ Зиганшин Малик Гарифович², \\ mjihan@mail.ru \\ 'О00 «Газпром трансгаз Казань», \\ Россия, 420073, г. Казань, ул. А. Кутуя, 41. \\ 2 Казанский государственный энергетический университет, \\ Россия, 420066, г. Казань, ул. Красносельская, 51.
}

\begin{abstract}
Актуальность исследования обусловлена необходимостью повышения энергетической и экологической эффективности систем очистки выбросов систем пылеприготовления и дымовых газов ТЭС при угольной генерации и систем подготовки газа при газовой генерации, во исполнение Федерального закона № 261-Ф3 от 23.11.2009 г. (ред. от 29.07.2017) "Об энергосбережении и о повышении энергетической эффективности и о внесении изменений в отдельные законодательные акты Российской Федерации», а также требований по контролю за выбросом парниковых газов (напр., приказа № 330 от 29.06.2017 года "Об утверждении методических указаний по количественному определению объёма косвенных энергетических выбросов парниковых газов» и др.) выбросов систем пылеприготовления и дымовых газов ТЭС с угольной генерацией и в системе подготовки газа с газовой генерацией.

Цель: разработка устройства циклонной фильтрации для повышения эффективности систем газоочистки на ТЭС, снижения выбросов взвешенных частиц в атмосферу от систем пылеприготовления и дымовых газов при угольной генерации и увеличения надежности работы агрегатов газотурбинных и парогазовых установок ТЭС вследствие снижения износа рабочих поверхностей поршневых и винтовых дожимающих компрессоров дожимных компрессорных станций, предотвращения попадания продуктов внутренней коррозии газопроводов в газотурбинных установках, в соответствии с п.П. 93, 94 ФНиП в области промышленной безопасности «Правила безопасности сетей газораспределения и газопотребления» (Серия 12, вып. 13).

Объект: модель циклона на базе ЦН-11-200, обеспечивающая повышение энергетической и экологической эффективности систем газоочистки на ТЭС.

Методы: стендовые испытания циклонного фильтра, изготовленного путем модернизации циклона ЦН-11-200, аэродинамические расчёты; численное моделирование аэродинамических параметров работы циклона-фильтра на основе методов вычислительной гидродинамики. Для математического моделирования процесса сепарации использована система уравнений, состоящая из осредненных по Рейнольдсу уравнений движения однофазного потока Навье-Стокса и уравнения движения частиц, основанного на законе Ньютона. Для определения эффективности отделения взвешенной части потока в циклоне-фильтре использован безразмерный комплекс $R e_{r}$ с п помощью которого численные значения параметров осаждения взвеси из многофазного потока в сепараторе найдены расчетным путем.

Результаты. Анализ литературных источников показал отсутствие конструкций циклонов-фильтров, которые обеспечивали бы надлежащую (более 98 \%) степень очистки выбросов без резкого увеличения энергозатрат на обработку выбросов. Проведенные опыты показали целесообразность конструктивного дополнения возвратно-поточных циклонов фильтрующей вставкой в зоне, где происходит инерционное осаждение пыли. Для определения эффективности отделения взвешенной части потока в данном циклоне-фильтре был использован безразмерный комплекс $R e_{r .}$ Расчеты показали соответствие результатов теоретических и натурных исследований.
\end{abstract}

Ключевые слова:

Циклон, фильтр, сепарация, степень очистки, метод моделирования, энергозатраты, парниковые газы.

\section{Введение}

Непрекращающийся рост мирового потребления энергии выдвинул в качестве одного из глобальных вызовов человечеству проблему энергогенерации в условиях сокращения невозобновляемого энергоресурса - ископаемых топлив, и роста загрязнения атмосферы планеты. Ее увязанное решение, включающее минимализацию как объемов выбросов, так и энергозатрат на их обработку, становится приоритетной целью современной отечественной и мировой энергетической политики. При этом уже сегодняшний уровень развития техники генерации позволяет существенно, почти вдвое, повысить эффективность использования ископаемого топлива на тепловых станциях, и пропорциональ- но уменьшить объемы атмосферных выбросов. С этим связан и наблюдаемый сейчас перевод генерации тепловой и электрической энергии на газотурбинные и парогазовые циклы, что дополнительно позволит несколько оптимизировать долю природного газа в топливном балансе ТЭС, которая сейчас находится на уровне порядка $70 \%$.

C позиции состояния атмосферы в крупных городах европейской части РФ такое положение может рассматриваться как благоприятное. Однако на сегодня базовые месторождения Западной Сибири, обеспечивающие основную часть текущей добычи, в значительной мере уже выработаны: Медвежье - на $78 \%$, Уренгойское - на $67 \%$, Ямбургское - на $46 \%$. Имея в виду еще новые эк- 
спортные направления по АТР (Азиатско-тихоокеанский регион), можно полагать, что переход с природного газа на чистую угольную генерацию может быть востребован в не столь отдаленной перспективе [1], даже с учетом освоения новых материковых и шельфовых месторождений газа.

Пока теплоэнергетические установки угольной генерации служат крупными поставщиками выбросов как с гетерогенными, так и с гомогенными загрязнителями. $\mathrm{K}$ примеру, от угольных электростанций в атмосферу планеты поступает за год порядка $200 \cdot 10^{6}$ т золы и $40 \cdot 10^{6}$ т диоксида серы. Поэтому вопросы энергосбережения и повышения экологической эффективности систем очистки продуктов сгорания на углесжигающих ТЭС очень актуальны и на сегодня сравнимы с проблемами достижения максимальной энергоэффективности при генерации.

Электростанции России и зарубежных стран, работающие на твердом топливе, имеют резервы совершенствования. В значительной степени это касается проблем оптимизации способов обработки выбросов с целью уменьшения попадания в атмосферу взвешенных частиц классов $\mathrm{PM}_{10}$ и, в особенности, $\mathrm{PM}_{2,5}$, в направлении снижения энергозатрат и повышения экологической эффективности.

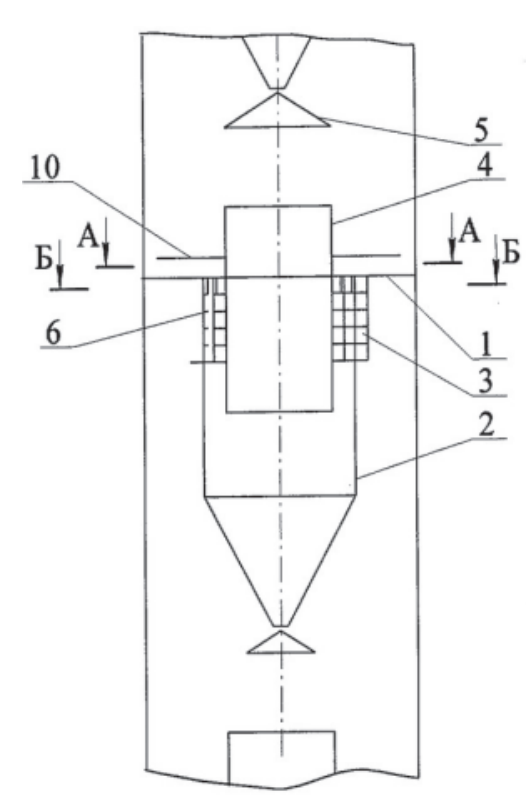

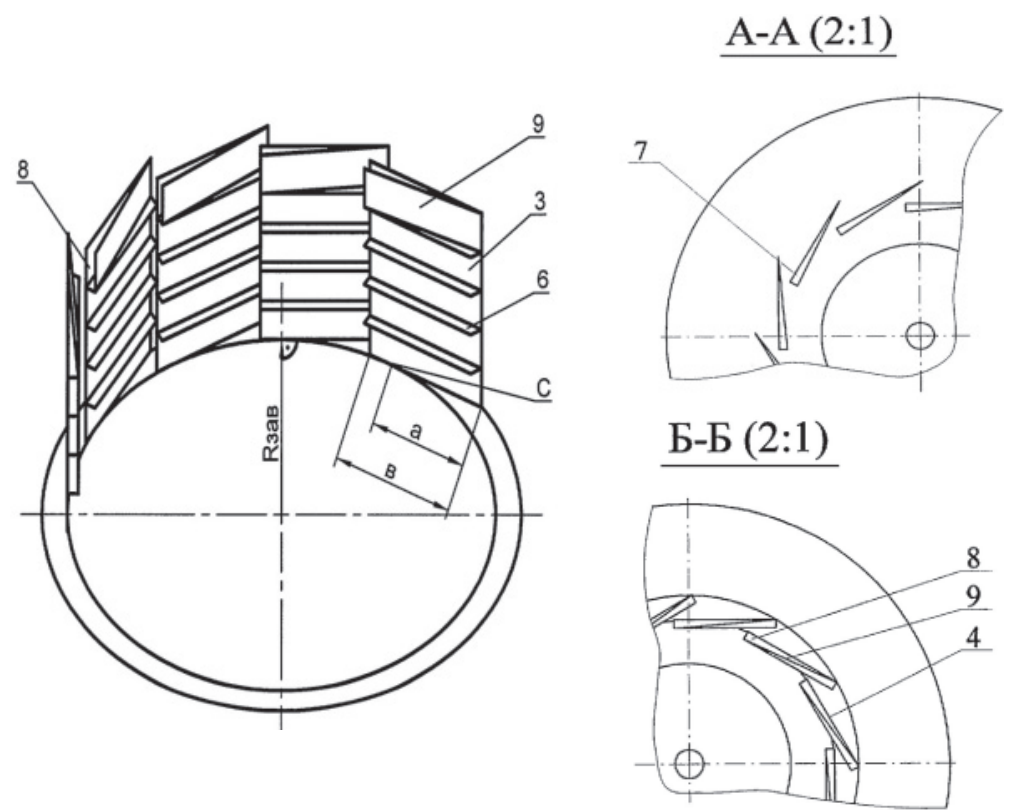

Анализ способов повышения эффективности сепарации в аппаратах, реализующих принцип циклонной фильтрации

Сейчас для пылезолоосаждения в производственных условиях широко применяются циклонные пылеуловители, что объясняется преимуществом ряда их технико-экономических показателей по сравнению с другими видами пылеуловителей. Это относится к таким показателям, как простота конструкции и изготовления, высокая пропускная способность на единицу массы оборудования, небольшие эксплуатационные расходы, возможность улавливания пыли в сухом виде, сохранение требуемой фракционной эффективности очистки при увеличении загрузки потока твердой фазой, надежность работы при достаточно высокой температуре и др. Поэтому в России и за рубежом интенсивно ведутся поиски путей совершенствования циклонных устройств в направлении повышения степени осаждения взвешенных частиц и снижения энергоемкости циклонной очистки. В работе [2], например, представлена конструкция вихревого аппарата с многоступенчатым контактом фаз (ВКУ). Попадая в аппарат, смесь газа и жидкости движется вниз до лопастей - 3 (рис. 1), где происходит их интенсивная закрутка. При осевом выходе газа из ВКУ вверх по трубе - 4 происходит се-

Рис. 1. Вихревой пылеуловитель для мокрой многоступенчатой очистки газовых выбросов (Патент № 2257248 РФ), где 1 - полотно; 2 - переливное устройство с завихрителем; 3 -лопасти, закрепленные верхней частью в полотне и заглушенных в нижней части, 4 - труба для выхода газа; 5 - отражательный диск; 6 - горизонтальные пластины; 7 - V-образные отверстия для подвода жидкости; 8-V-образный канал; 9 - вертикальная пластина; 10 - фальшдно; $R_{\text {зав }}$ - радиус завихрителя; $а$ - ширина лопасти от ее начала на внешнем диаметре завихрителя до точки ее касания с внутренним диаметром завихрителя; в - общая ширина лопасти; с-точка касания лопасти кокружности

Fig. 1. Vortex dust collector for wet multistage cleaning of gas emissions (Patent № 2257248 of the RF), where 1 is the cloth; 2 is the overflow device with a vortex generator; 3 are the blades fixed by the top part in a cloth and muffled in the lower part; 4 is the pipe for gas discharge; 5 is the reflective disk; 6 are the horizontal plates; 7 are the $V$-shaped openings for liquid supply; 8 is the V-shaped channel; 9 is the vertical plate; 10 is the false bottom; $R_{3 а в}$ is the swirl radius; $a$ is the width of the blade from its beginning on the outer diameter of the swirl to the point of contact with the inner diameter of the swirl; 8 is the general blade width; $c$ is the point of contact of the blade to the circle 
парация взвеси. Равномерность скорости газа достигается за счет изменения зазора между лопатками. Для повышения удерживающей способности по жидкости на лопатках завихрителя имеются дополнительные горизонтальные пластины - 6 . Преимущество предлагаемого тепломассообменного устройства заключается в повышении эффективности сепарации вследствие увеличения удерживающей способности за счет горизонтальных пластин. Недостатками указанного аппарата являются существенные энергозатраты из-за повышенного гидравлического сопротивления завихрителя и высокий брызгоунос.

Авторами работы [3] представлен HL-циклон высокой производительности (Hochleistungszyklon). Он отличается от стандартных классических циклонов сквозной центральной («направляющей») трубой в оси циклона, сильно зауженным входным патрубком запыленного газа, существенно укороченной конической частью циклона, впадающей в бункер. По мнению авторов разработки, конструктивное дополнение в виде центральной трубы, расположенной по оси HL-циклона (рис. 2), обеспечивает высокоэффективную работу аппарата данной конструкции. По представлениям авторов [4], в классических стандартных циклонах без центральной направляющей трубы переход от ламинарного к турбулентному течению происходит внутри выхлопной трубы аппарата. В результате ядро вихря потока в аппарате становится нестабильным, что вызывает большие потери давления и энергозатраты, по-видимому, на перестройку структуры потока, заключающуюся в разрушении организованного вращательного движения потока с образованием мелких вихрей стохастического характера. По [5] это вызывает, в свою очередь, высокие потери (до $90 \%$ от общей потери давления) вследствие диссипации энергии в потоке. Авторы [6] заключают, что роль центральной («направляющей») трубы на оси цикло- на заключается в стабилизации вращающегося течения потока и в сдвиге перехода от ламинарного к турбулентному режиму в область более высоких чисел Рейнольдса.

В этом случае потеря статического давления $\Delta P_{\text {stat }}$ снижается, а степень разделения улучшается ввиду увеличения динамического напора. Однако опыты на конструкции HL-циклона проводились авторами при нагрузках в $1,5 . .2$ раза ниже оптимальных для классических стандартных циклонов такого типоразмера. Следовательно, испытанные режимы могут не соответствовать условиям промышленной эксплуатации аппаратов, и эффективность, достигнутая в экспериментах, в реальных условиях может не подтвердиться.

Для очистки производственных выбросов, в том числе загрязненных особо опасными взвесями, а также при необходимости тонкой очистки и обеззараживания вентиляционного воздуха, подаваемого, например, в операционные отделения учреждений здравоохранения, может применяться циклон-фильтр [7] (рис. 3).

Техническая задача интенсификации циклонной обработки и повышения степени осаждения тонкодисперсных частиц решается здесь путем увеличения скорости дисперсного потока с одновременным принятием мер по предотвращению абразивного износа внутренних поверхностей аппарата. Устройство позволяет уменьшить ресурсоемкость, однако энергозатраты растут пропорционально увеличению скорости.

Анализ множества других существующих устройств циклонной фильтрации производственных дисперсных выбросов [8-11] также показал, что устройства, обладающие высокой степенью осаждения частиц классов $\mathrm{PM}_{10}$ и $\mathrm{PM}_{2,5}$, одновременно являются материало- и энергозатратными. Аппараты же с низким сопротивлением и, соответственно, энергоэффективные, имеют невысокую степень осаждения частиц указанных классов.

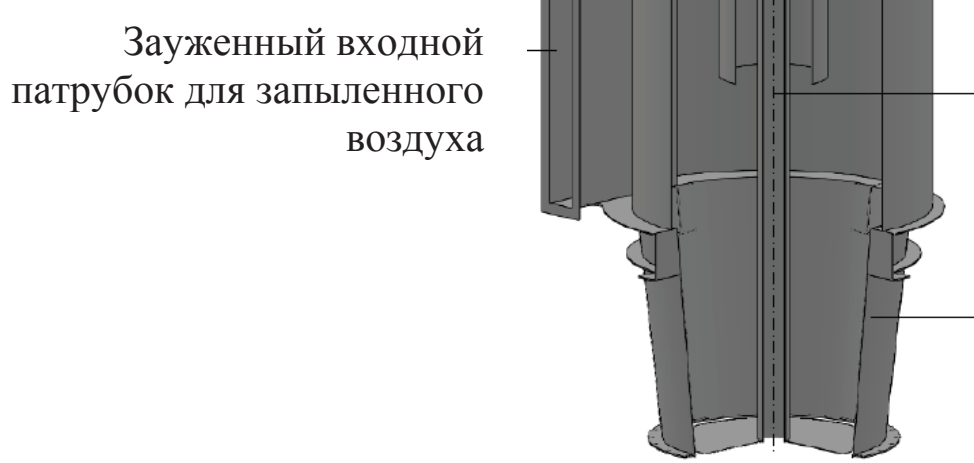

Зауженный входной воздуха

Puc. 2. Конструкция HL-ииклона [3]

Fig. 2. HL-cyclone design [3]
Сквозная центральная труба

\section{Укороченная коническая} часть циклона 


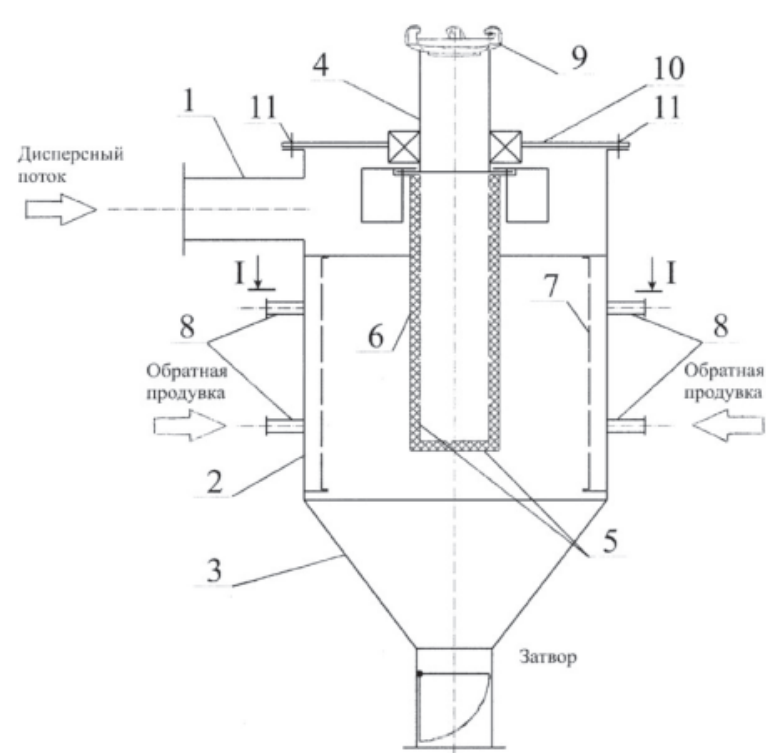

Рис. 3.Циклон-фильтр (патент № 2361678 РФ) [7]: 1 - вход ной патрубок; 2 - корпус; 3 - коническое днище; 4 - шту uер отвода; 5 - иилиндричекий каркас; 6 - плотный фильтровальный материал; 7 - тонкая фильтровальная ткань; 8 - продувочные штуцеры; 9 - цапфовый элемент; 10 - верхний тореи; 11 - крепежные әлементы разьема

Fig. 3. Cyclone filter (Patent No. 2361678 RF) [7]: 1 is the inlet; 2 is the body; 3 is the conical bottom; 4 is the fitting challenge; 5 is the cylindricity frame; 6 is the dense filtering material; 7 is the thin filter fabric; 8 are the purge fittings; 9 is the pin element; 10 is the upper end; 11 are the retaining elements of the connector

\section{Циклонный фильтрующий аппарат \\ для повышения энергетической и экологической эффективности очистки}

С целью обеспечения надлежащей очистки газов от взвешенных частиц классов $\mathrm{PM}_{10}, \mathrm{PM}_{2,5}$ без увеличения энергозатрат на осаждение взвеси предложена усовершенствованная конструкция циклона-фильтра. Увеличение степени очистки направлено на достижение надежной работы агрегатов газотурбинных и парогазовых циклов ТЭС при газовой генерации и повышение энергоэффективности работы газоочистных устройств при угольной генерации. Так, например, повышение эффективности выделения взвешенной части из потока природного газа в пункте подготовки газа (ППГ) ТЭС приводит к снижению износа цилиндровых стенок поршневых компрессоров или поверхностей винтов и корпуса сложной конфигурации (точность обработки которых составляет 10 мкм) винтовых дожимающих компрессоров ДКС. Предлагаемая конструкция циклона-фильтра также может быть использована при угольной генерации для повышения степени очистки атмосферных выбросов систем пылеприготовления от угольной пыли и дымовых газов ТЭС от золы мелкодисперсных частиц классов $\mathrm{PM}_{10}, \mathrm{PM}_{2.5}$. Известно [12], что в летучей золе электростанций содержание таких частиц в среднем около $30 \%$, а в вы- бросах угольной и сланцевой пыли около - $10 \%$, и может достигать $35 \%$.

Предлагаемый циклонный фильтр (рис. 4) с тангенциальным подводом воздуха, совмещающий две ступени очистки, обеспечивает высокую энергетическую и экологическую эффективность очистки выбросов, содержащих частицы классов $\mathrm{PM}_{10}$ и $\mathrm{PM}_{2,5}$, при приемлемых энергозатратах и невысоком абразивном износе в реальных производственных условия. Для эффективного осаждения мелкодисперсных частиц с минимальными энергетическими и материальными затратами циклон имеет дополнительное устройство - вставку из тканого фильтра.

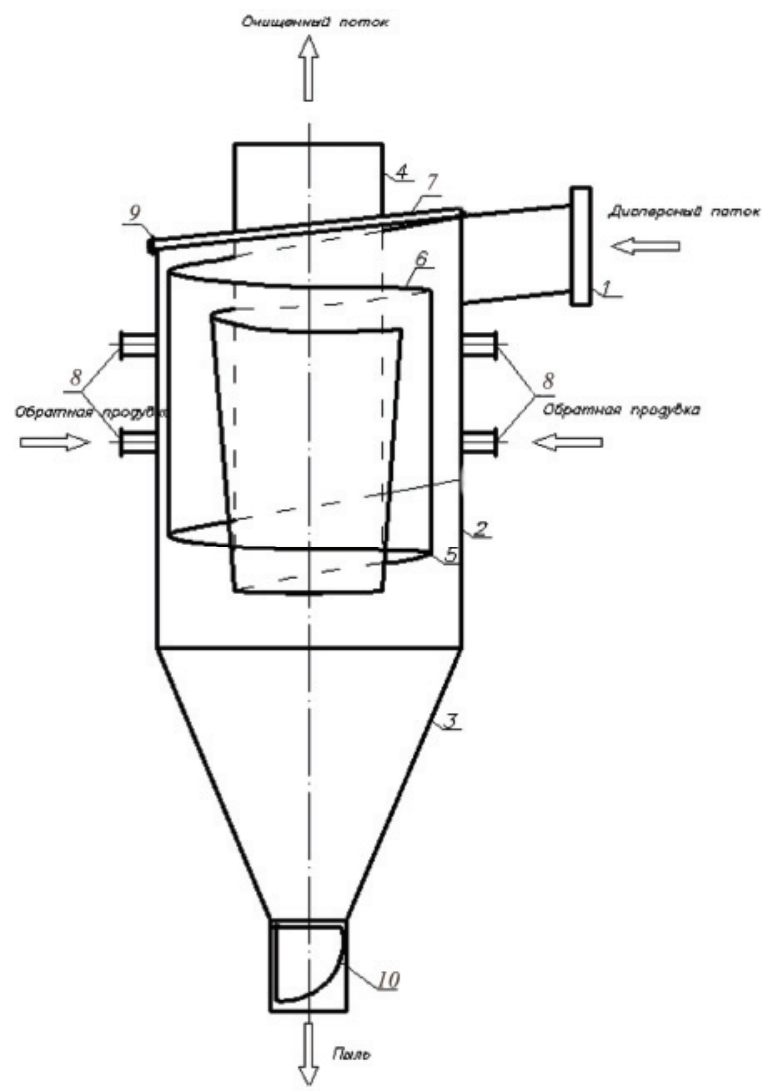

Pис. 4. Схема конструкиии ииклона-фильтра: 1 - входной патрубок; 2 - иилиндрический корпус; 3 - коническое днище; 4 - выхлопная труба; 5 - фильтровальный материал; 6 - металлический каркас; 7 - тореи ииклона; 8 продувочные шттуцера; 9 - детали крепежа; 10 - заслон$\kappa a$

Fig. 4. Scheme of a design of a cyclone filter: 1 is the entrance branch pipe; 2 is the cylindrical body; 3 is the conic bottom; 4 is the exhaust pipe; 5 is the filtering material; 6 is the metal framework; 7 is the end face of a cyclone; 8 is the blowing-off union; 9 are the parts of fixture; 10 is the valve

Циклон-фильтр содержит штуцер для ввода запыленного газового потока - 1 , установленный тангенциально на цилиндрическом корпусе - 2 , имеющем коническое днище - 3 с затвором - 10, расположенную соосно с цилиндрическим корпусом выхлопную трубу -4 , по диаметру которой внутри корпуса циклона установлен фильтровальный материал - 5, (например, лавсан), зафиксированный в 
металлическом каркасе - 6, который закреплен к торцу циклона - 7. Снаружи, радиально цилиндрического корпуса - 2, установлены продувочные штуцера -8 , также имеются детали - 9 крепежа цилиндрического корпуса для разъемного торца, в случае замены каркаса с фильтром.

На базе данного серийного циклона ЦН-11 диаметром 200 мм проведены стендовые испытания циклона-фильтра, конструкция которого была разработана авторами. Испытания проводились на специально приготовленном дисперсном материале - шамотной пыли с размером частиц до 63 мкм. Пробу материала для испытаний отбирали в соответствии с надлежащей лабораторной практикой по ГОСТ Р 51568-99 (ИСО 3310-1-90). Радиус частиц по результатам седиментационного анализа по фракциям получился от 1,23 до 31,51 мкм; следовательно, наибольший диаметр частиц пыли по результатам седиментационного анализа равен 63 мкм. Дисперсный состав пыли в выбросах после циклона-фильтра определялся при помощи импактора. Результаты анализа дисперсного состава потока показали, что среднемедианный размер частиц $d_{50}=1,6$ мкм (таблица).

таблица. Фракционный состав пыли

Table. Fractional composition of dust

\begin{tabular}{|l|c|c|c|c|c|c|}
\hline $\begin{array}{l}\text { Paзмер фракции, } \text { м }^{-6} \\
\text { Particle size, } \mathrm{m}^{-6}\end{array}$ & $>0,4$ & $>0,8$ & $>1,6$ & $>3,5$ & $>6,0$ & $>10$ \\
\hline $\begin{array}{l}\text { Мacсовая доля фракции, \% } \\
\text { Mass fraction, \% }\end{array}$ & 80 & 70 & 50,0 & 30 & 10 & 5 \\
\hline
\end{tabular}

Образцы фильтровального материала взвешивались в начале и конце испытания. После окончания испытания с начальной, средней и конечной частей фильтровальной ткани вырезались образцы для сравнительного определения эффективности осаждения по счетной концентрации уловленных частиц, которая устанавливалась в результате изучения образцов под микроскопом МИН-8 с 400-кратным увеличением. Это позволило провести определение счетного количества частиц классов $\mathrm{PM}_{2,5}, \mathrm{PM}_{10}$, что необходимо для достоверного сопоставления интенсивности их оседания из потока на фильтр при различных режимах. Результаты испытаний показали возможность повышения эффективности осаждения частиц указанных классов при приемлемых энергозатратах.

Проведенные опытные исследования подтвердили целесообразность конструктивного дополнения возвратно-поточных циклонов фильтрующей вставкой в зоне, где происходит инерционное осаждение пыли. Вместе с тем, с целью доработки конструкции циклона-фильтра, был выполнен ряд исследований на базе расчетных методов. Для определения эффективности отделения взвешенной части потока в данном циклоне-фильтре был использован комплекс $\operatorname{Re}_{r}[13]$ :

$$
\operatorname{Re}_{r}=\frac{U_{0} \rho_{p}^{2} D_{p}^{4}}{c \rho_{g} R_{2}^{3} \eta},
$$

где $U_{0}$ - начальная скорость, м/с; $\rho_{p}$ - плотность частиц, кг $/ \mathrm{M}^{3} ; D_{p}$ - диаметр микрочастиц, м; $c$ - коэффициент, зависящий от соотношения радиусов внутренней и внешней границ кольцевого канала завихрителя (у большинства отечественных серийных возвратно-поточных циклонов это соотношение диаметров корпуса и выхлопной трубы, равное $0,59$, для которого $c=27) ; \rho_{g}-$ плотность газа, $\kappa \Gamma / \mathrm{M}^{3} ; R_{2}$ - характерный радиус криволинейной траектории для движущейся в конкретных условия частицы, за который в циклоне принимается радиус корпуса циклона, м; $\eta$ - коэффициент динамической вязкости, Па.с.

Параметр $\mathrm{Re}_{r}$ получен в результате использования двух общепринятых методов теории подобия анализа размерностей и масштабирования, с приведением системы уравнений, состоящих из уравнений Навье-Стокса для движения потока и закона Ньютона для движения частицы, к безразмерному виду. Для приведения размерных переменных величин в них использованы в качестве масштабов радиус аппарата и начальная скорость входа в него гетерогенного потока. В литературе для определения степени осаждения взвешенных частиц из потоков часто рекомендуется использование числа Стокса. Одним специфичным отличием параметра $\mathrm{Re}_{r}$ от числа Стокса является то, что геометрическое масштабирование произведено не по линейной величине - размеру препятствия, а по радиусу кривизны $R_{2}$ линии тока, обходящей препятствие. Другое отличие в том, что инерционным масштабом служит не масса, а момент инерции, в связи с чем здесь полнее отражается специфика рассматриваемой конкретной системы, заключающаяся в описании сложного криволинейного движения частицы возле препятствия с поворотом как по траектории, так и вокруг своей оси. Таким образом, число $\mathrm{Re}_{r}$ содержит безразмерные инерционный $J_{p} / J_{G}$ и геометрический $r_{p} / R_{2}$ параметры частицы, которые более полно, чем число Стокса, характеризуют движение частицы по криволинейной траектории. Более широкое обсуждение данного вопроса со сравнительной характеристикой диапазонов размеров частиц, в которых приемлемы расчеты с использованием чисел Stk и $\mathrm{Re}_{r}$, coдержится в работах $[14,15]$. Безразмерный комплекс $\operatorname{Re}_{r}$ имеет структуру критерия Рейнольдса и составлен посредством комбинации параметров, выражающих соотношение энергий и действий частицы и вращающегося потока. Поэтому в криволинейных (вращающихся, вихревых) потоках численные значения критерия $\operatorname{Re}_{r}$ по (1) должны соответствовать степени осаждения частиц пыли и могут использоваться для ее оценки в сходственных условиях [16].

Прослеживается также зависимость от энергетических затрат: $\operatorname{Re}_{r} 99$ уменьшается с увеличением сопротивления аппарата. Следует заметить, что в структуру $\operatorname{Re}_{r}$ коэффициент гидравлического сопротивления циклона не входит. Это выражение позволяет легко определять минимальную ско- 
рость потока и соответствующие затраты энергии, обеспечивающие требуемый процент осаждения частиц данного размера в исследуемом аппарате.

Результаты расчетов значений $\mathrm{Re}_{r}$ для гетерогенного потока в модели, соответствующей по размерам циклону-фильтру на базе ЦН-11-200 и при соответствующих опыту рабочих условиях, для размеров частиц $D_{p}=(1 . .100) \cdot 10^{-6}$ м и относительной скорости потока на входе в циклон $U_{0}=1,0 . . .14,0$ м/с, представлены на рис. 5 .

Расчеты показали, что степеням осаждения частиц в циклоне соответствуют строго определенные значения чисел $\mathrm{Re}_{r}$. Так, степени осаждения 99 \% при скорости 5,0 м/с может быть сопоставлено число $\operatorname{Re}_{r}=2,4 \cdot 10^{-3}$, а степени осаждения $50 \%$ $\mathrm{Re}_{r}=4,65 \cdot 10^{-8}$. Проведенные исследования показывают, что параметр $\operatorname{Re}_{r}$ позволяет находить численные значения параметров очистки в сепараторах с вращательным движением многофазных потоков расчетным путем. Расчеты на основе $\operatorname{Re}_{r}$ приводят к результатам, близким к опытным. Параметр $\mathrm{Re}_{r}$ позволяет устанавливать режимы инерционной сепарации частиц различных размеров из потоков с криволинейными линиями тока, что характерно для потоков и в циклонах, и в пористой среде.

Вместе с тем частицы взвеси, сталкиваясь в потоке воздуха, могут подвергаться изменению размеров вследствие коагуляции или разрушения еще до очистного устройства. Это стохастические процессы, результаты которых зависят от огромного числа факторов, в том числе от физико-химических свойств взвесей (дисперсный состав, агрегатное состояние, увлажненность, УЭС, слипаемость, поверхностное натяжение, упругость, плотность, прочность, пористость и т. п.), несущей среды (влажность, температура, вязкость, плотность и др.). Поэтому результаты, основанные на теоретических моделях, всегда нуждаются в эмпирических корректировках, а на практике расчет степени осаждения взвешенных частиц ведется на их медианный (средний) диаметр. Однако если размеры частиц конкретного вида взвеси вследствие коагуляции или диспергации в конкретном потоке перед циклоном-фильтром окажутся известными, при помощи числа $\mathrm{Re}_{r}$ несложно вычислить степень осаждения частиц с учетом произошедших до пористого фильтра процессов, поскольку оно позволяет рассчитывать степень осаждения частиц любого размера.

Существенную роль в процессе фильтрационной очистки гетерогенных выбросов от твердофазных взвесей играют также процессы удержания оседающих частиц на элементах пористого слоя, образования автослоя и регенерации основы. Учет влияния этих процессов на эффективность фильтрации может быть осуществлен по разработанной одним из авторов настоящей статьи полуэмпирической модели фильтрации на тканом материале $[14,16]$, в основе которой также находится параметр $\operatorname{Re}_{r}$. В указанной модели параметр $\mathrm{Re}_{r}$ позволяет определить кинетическую энергию частиц, движущихся по криволинейным траекториям перед препятствиями, составляющими пористый слой. Как отмечалось ранее, параметр $\operatorname{Re}_{r}$ позволяет определять режим инерционной сепарации из потоков с криволинейными линиями тока, что имеет место и в циклонах, и в пористой среде. Это служит физической основой для принципиальной возможности учитывать также с помощью критерия $\mathrm{Re}_{r}$ перераспределения эффективностей инерционной сепарации частиц между фильтром и циклоном. Конкретно повышенная по сравнению с циклоном степень осаждения частиц в пористом слое отразится непосредственно в формуле (1) следующим образом. При фильтрации в пористом слое за величину $\mathrm{R}_{2}$ вместо радиуса циклона принимается радиус поворота частиц перед препятствием, который в этом случае так же, как и радиус циклона для потока в циклоне, определяет характерные для криволинейной траектории безраз-
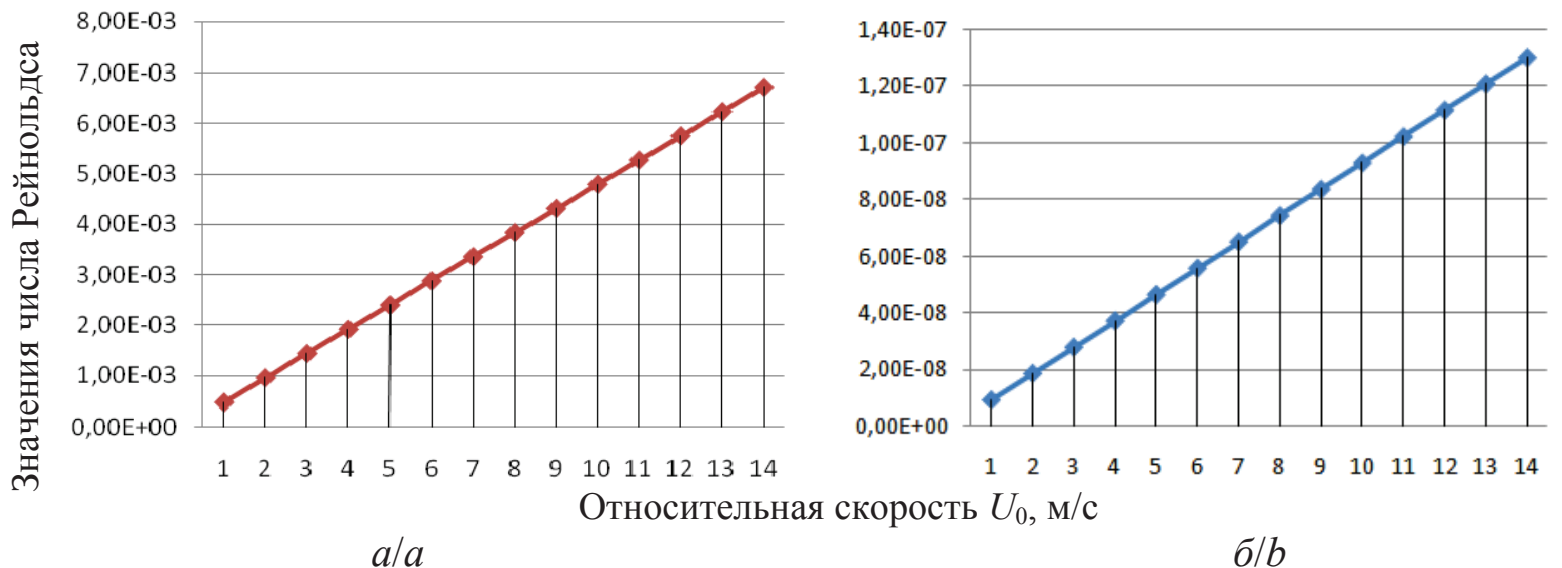

Puc. 5. Зависимость безразлерного числа $R e_{r}$ для ииклона с радиусом $R=0,1$ м от относительной скорости потока на входе в ииклон $U_{0}$, м/с для частии, оседающих на: а) $99 \%$; б) $50 \%$

Fig. 5. Dependence of relative number $R_{r}$ for a cyclone with radius $R=0,1 \mathrm{~m}$ on relative speed of a stream at the entrance to the $U_{0}$ cyclone, $m / s$ for particles settling for: a) $99 \%$; b) $50 \%$ 
мерные параметры взвешенных частиц - инерционный $J_{p} / J_{G}$ и геометрический $r_{p} / R$. Вопросы определения величин $R_{2}$ радиусов поворота взвешенных частиц перед препятствиями подробно рассмотрены в ряде публикаций авторов данной статьи, например в [17], там же приведены и эмпирические формулы для их нахождения.

$\mathrm{B}$ дальнейшем число $\mathrm{Re}_{r}$ сопоставляется с показателем энергии межмолекулярного взаимодействия поверхностных элементов пористого слоя и осаждающихся частиц в виде безразмерного энергетического параметра действия потока на них $A_{p}^{\text {rot }}$, ответственного за их удержание на препятствии [16].

Также было выполнено численное моделирование аэродинамических параметров работы циклона-фильтра на основе методов вычислительной гидродинамики. На сегодняшний день существует множество работ, посвященных моделированию запыленных потоков в циклонах на основе CFD (Computational Fluid Dynamics) [18-20]. Однако в них наблюдались только определенные характеристики потока в конкретных модификациях циклонов. Поэтому были проведены численные исследования 3d-модели потока в разработанной конструкции циклона-фильтра.
В условиях, когда энергетические параметры вращающегося в аппарате гетерогенного потока соизмеримы с величиной его входной энергии, для описания процесса сепарации целесообразно использовать систему из осредненных по Рейнольдсу уравнений Навье-Стокса движения однофазного потока и уравнения движения частиц, основанного на законе Ньютона. Для обеспечения замкнутости системы в уравнениях переноса и потери (диссипации) кинетической энергии была использована модель Спаларта-Аллмараса. Ее математическая формулировка достаточно полно рассмотрена в литературе, например, в работах [21-23].

На рис. 6 представлены эпюры тангенциальной скорости потока в двух поперечных сечениях и показаны расположения изотах в продольном сечении модели при входной скорости 5,0 м/с. После тангенциального входа поток газа приобретает в корпусе циклона осесимметричное винтовое движение. В продольном сечении 1-1 после кольцевой зоны скорость потока при перемещении вниз начинает расти в сужающейся конической части циклона и достигает 23,0 м/с. В поперечных сечениях 2-2 наблюдается увеличение тангенциальной составляющей скорости по радиусу по мере удаления от стенок циклона - в сечении 2-2 до 5,5 м/с, в сечении 3-3 до 10,5 м/с.

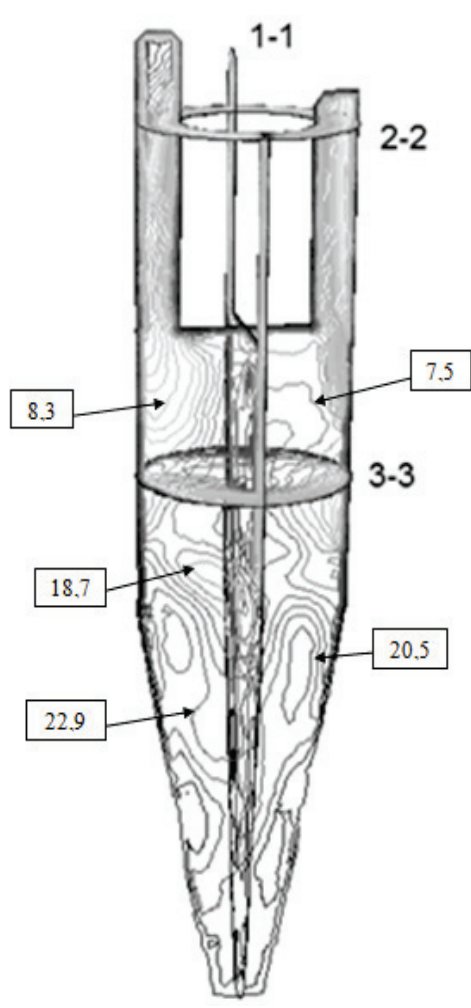

a

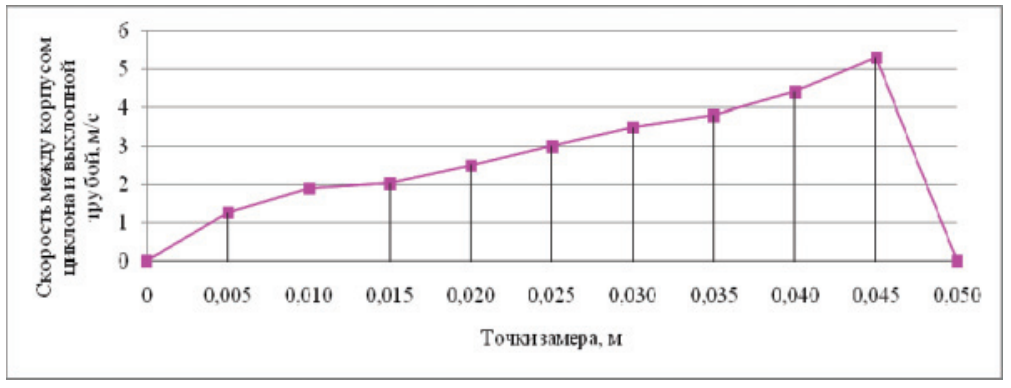

$b$

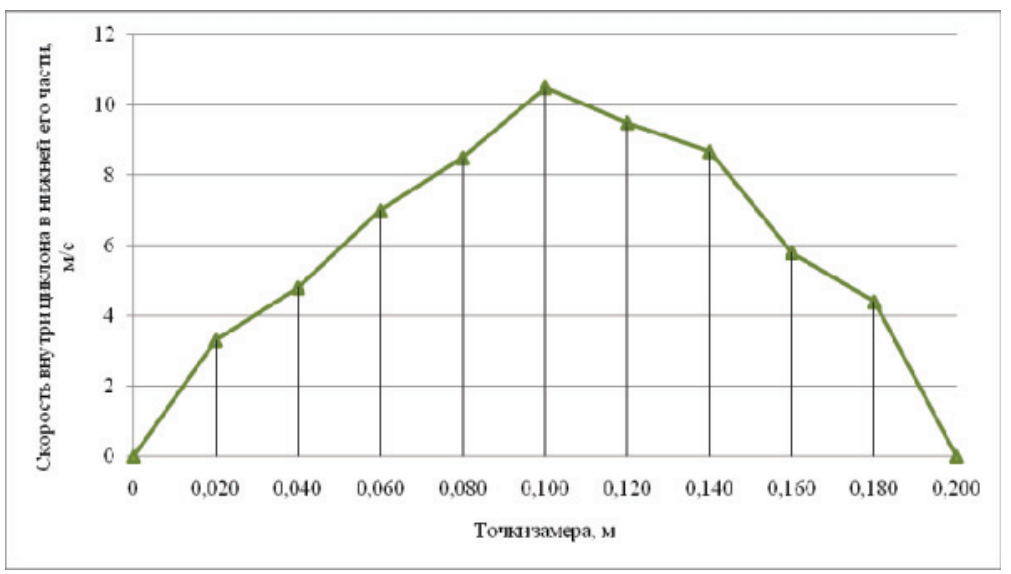

c

Puc. 6. Распределение тангенииальной скорости потока внутри циклона: а) в продольном сечении 1-1 (в поле рисунка указаны значения изотах в м/с); b) в поперечном сечении 2-2 от стенки корпуса (начало координат) до стенки выхлопной трубы; c) в поперечном сечении 3-3 между стенками корпуса

Fig. 6. Tangential flow rate distribution inside the cyclone: a) in the longitudinal section 1-1 (in the figure field are the values of the isotaxs in $\mathrm{m} / \mathrm{s}$ ); $b$ ) in the cross section 2-2 from the housing wall (origin) to the wall of the exhaust pipe; $c$ ) in the cross section 3-3 between the walls of the housing 
В соответствии с известной энергетической концепцией улавливания взвешенных частиц из гетерогенного потока, степень их осаждения пропорциональна величине энергетических затрат, непосредственно связанных с процессами осаждения. В конструкции представленного циклона-фильтра это процессы осаждения на поверхностях фильтровальной вставки и внутренней стенки циклона. Потери же энергии на сопротивления, возникающие при движении запыленного потока в соединительных фасонных деталях перед циклоном и при его входе в циклон, таковыми не являются, но они неизбежны в процессе эксплуатации аппарата.

Проведенные численные исследования показали, что для снижения указанных потерь могут использоваться профилирующие вставки для каналов с боковыми отверстиями, формы профилей которых также получены на основе компьютерного моделирования течений методами CFD [24-28].

С учетом этого представленный в данной работе циклон-фильтр позволяет увеличить пропускную способность очистных аппаратов в 4 раза при повышении качества очистки газа, которое выража-

\section{СПИСОК ЛИТЕРАТУРЫ}

1. Крупнейшие месторождения газа в России. URL: http://vivareit.ru/krupnejshie-mestorozhdeniya-gaza-v-rossii (дата обращения 04.04.2019).

2. Хамидуллин Р.Н. Технология очистки газовых выбросов от пыли производства силикатного кирпича: автореф. дис... канд. тех. наук. - Казань, 2005. -20 с.

3. Brunnmair E. Entwicklung und Modellierung eines neuen Hochleistungszyklons zur Trennung von Feststoff/Gas-Gemischen: Diss. ... Doktors der montanistischen Wissenschaften. Leoben, 2015. - $168 \mathrm{p}$.

4. Bielefeldt E.A. Wirbelkammern neurer Technologie // Journal of Professional Issues in Engineering Education and Practice. 2013. - V.15. - P. 286-291.

5. Muschelknautz E. Theorie der Fliehkraftabscheider mit besonderer Berücksichtigung hoher Temperaturen und Drücke // VDI-Bericht Nr. - 1980. - № 383. - P. 49-60.

6. Staudinger G. Zyklone mit Zentralkörpern Veröffentlichung // Chem. Ing.-Tech. Journal. - 1992. - V.64. - P. 769-888.

7. Циклон-фильтр: пат. 2361678 Российская Федерация: МПК B04C9/00, B01D50/00; заявл. 14.03.2008; опубл: 20.07.2009. Бюл. № 20. -5 c.

8. An alternative for the collection of small particles in cyclones: experimental analysis and CFD modeling / E. Balestrin, R.K. Decker, D. Noriler, J.C.S.C. Bastos, H.F. Meier // Separation and Purification Technology. - 2017. - № 5. - P. 54-65.

9. Increasing Efficiency of Natural Gas Cyclones through Addition of Tangential Chambers / W.I. Mazyan, A. Ahmadi, H. Ahmed, M. Hoorfar // Journal of Aerosol Science. - 2017. - V. 4. № 11. - P. $36-42$.

10. Темникова Е.Ю., Богомолов А.Р., Петрик П.Т. Исследование характеристик циклона с внутренними элементами // Вестник Кузбасского гос. тех. унив. - 2009. -№ 2. - С. 140-144.

11. Серебрянский Д.А., Захаров А.А., Плашихин С.В. Циклонные пылеуловители. Малозатратная модернизация // Хімічна промисловість України. - 2013. - V. 3. - С. 70-74.

12. Атлас промышленных пылей. Ч. 1. Летучая зола промышленных электростанций / под ред. Л.Я. Скрябина. - М.: ЦИНТИХимнефтемаш. $-1980 .-48$ с. ется в уменьшении размера частиц, улавливаемых на $50 \%$ (диаметра отсекания), со средних для циклонов значений 5-10 до 0,4 мкм.

Указанное улучшение качества очистки не требует дополнительной затраты энергии, что является преимуществом перед аналогами. Энерго- и топливоресурсосбережение, в свою очередь, - это единственный на сегодняшний день реальный путь снижения выбросов парниковых газов, что соответствует обязательствам, принятым Российской Федерацией по Парижскому соглашению об изменении климата.

\section{Заключение}

По результатам натурных испытаний и исследований на базе расчетных методов проведена доработка конструкции циклона-фильтра с целью достижения высокой степени улавливания взвешенных частиц классов $\mathrm{PM}_{2,5}, \mathrm{PM}_{10}$ при приемлемых энергетических затратах на газоочистное оборудование в целом, включающих и пониженные гидравлические потери на транспортировку выбросов к очистному устройству.

13. Зиганшин М.Г., Зиганшин А.М. Лабораторно-практический курс пылегазоочистки. Ч. 1. - Казань: Изд-во КГАСУ. 2012. $-188 \mathrm{c}$.

14. Зиганшин М.Г., Зиганшин А.М. Полуэмпирическая модель образования автослоя на тканых фильтрационных материалах. Ч. 2. Опытное исследование фильтрации // Известия Казанского государственного архитектурно-строительного университета. - 2009. - V. 1. - № 11. - С. 186-190.

15. Еремкин А.И., Зиганшин М.Г. Степень инерционного осаждения взвешенных частиц в циклонных и вихревых сепараторах. Верификация параметров эффективности // Научный вестник Воронежского государственного архитектурно-строительного университета. - 2009. - V. 3. - № 15. - С. 137-145.

16. Зиганшин М.Г. Теоретические основы пылегазоочистки. - Казань: Изд-во КГАСУ. - 2005. - 262 с.

17. Зиганшин М.Г., Колесник А.А., Зиганшин А.М. Проектирование аппаратов пылегазоочистки. - СПб.: Лань. - 2014. - 544 с.

18. Fassani F.L., Goldstein L. A study of the effect of high inlet solids loading on a cyclone separator pressure drop and collection efficiency // Powder Technology. - 2000. - V.3. - № 18. - P. 60-65.

19. Effects of geometry and solid loading on the performance of gas cyclones / C. Hoffmann, A. Vansanten, R.W.K. Allen, R. Clift // Powder Technology. - 1992. - № 13. - P. 83-91.

20. CFD-DEM modeling of multiphase flow in dense medium cyclones / K.W. Chu, B. Wang, A.B. Yu, A. Vince // Powder Technology. 2009. - V. 1. - № 7. - P. 235-247.

21. Numerical Study of the Effect of Changing the Cyclone Cone Length on the Gas Flow Field / 0. Hamdy, M.A. Bassily, H.M. ElBatsh, T.A. Mekhail // Applied Mathematical Modelling. 2017. - V. 1. - № 26. - P. 81-97.

22. Modeling the multiphase flow in a dense medium cyclone / B. Wang, K.W. Chu, A.B. Yu, A. Vince // Industrial \& Engineering Chemistry Research. - 2009. - № 48. - P. 3628-3639.

23. Assessments of CFD-DEM models in particle fluid flow modeling / Z.Y. Zhou, S.B. Kuang, K.W. Chu, A.B. Yu // Journal of Fluid Mechanics. - 2010, - № 661. - P. 482-510.

24. Зиганшин А.М., Гимадиева Г.А., Батрова К.Э. Численное исследование истечения из бокового последнего и среднего отверстий // Сб. статей I Всероссийской научно-технической конфе- 
ренции, посвящённой памяти д-ра техн. наук, профессора А.А. Сандера. - Новосибирск: НГАСУ. - 2017. - С. 103-111.

25. Зиганшин A.M. Снижение энергозатрат при движении потоков путем профилирования фасонных частей в коммуникациях энергоустановок // Надежность и безопасность энергетики. $-2015 .-$ V.1 - № 28. - С. 63-68.

26. Соединительный фасонный элемент с профилирующими вставками: пат. 2604264 Российская Федерация: МПК F16L 43/00, МПК F16L 25/14; заявл. 17.09.2014; опубл. 10.12.2016. Бюл. № 34. -13 c.
27. Зиганшин А.М., Гимадиева Г.А., Батрова К.Э. Потери давления и характеристики струи, выходящей через среднее боковое отверстие // Известия КазГАСУ. - 2017. - V.4 - № 42. C. $257-265$.

28. A survey of separated airflow patterns at inlet of circular exhaust hoods / K.I. Logachev, A.M. Ziganshin, O.A. Averkova, A.K. Logachev // Energy \& Buildings. - 2018. - № 173. - P. 58-70.

Поступила 27.08.2019 2.

\section{Информация об авторах}

Замалиева A.T., инженер 000 «Газпром трансгаз Казань».

Зиганшин М.Г., доктор технических наук, доцент кафедры тепловых электрических станций Казанского государственного энергетического университета. 
UDC 621.928.9

\title{
IMPROVE ENERGY AND ENVIRONMENTAL EFFICIENCY OF GAS TREATMENT SYSTEMS ON TPP
}

\author{
Albina T. Zamalieva', \\ Albina-0587@rambler.ru \\ Malik G. Ziganshin², \\ mjihan@mail.ru \\ 1 LLC «Gazprom transgaz Kazan»,
41, A. Kutuy street, Kazan, 420073, Russia. \\ 2 Kazan State Energy University, \\ 51, Krasnoselsky street, Kazan, 420066, Russia.
}

\begin{abstract}
The relevance of the research is caused by the necessity to increase energy and environmental efficiency systems for purification of emissions of pulverizing system and flue gas thermal power plant in coal generation and gas treatment in gas generation, pursuant to Federal law No. 261-FZ dated 23.11.2009 (ed. from 29.07.2017) "On energy saving and increasing energy efficiency and amendments to certain legislative acts of the Russian Federation" and the requirements to control the emission of greenhouse gases (e.g., order No. 330 of 29.06.2017 "On approval of methodical instructions for quantitative determination of the amount of indirect energy greenhouse gas emissions», etc) emissions of pulverizing system and flue gases of thermal power plants with coal generation and the system gas treatment gas generation.

The main aim of the research is to develop a cyclone filtration device to increase the efficiency of gas treatment systems at thermal power plants, reduce suspended particulate emissions into the atmosphere from dust preparation systems and flue gases during coal generation and increase the reliability of gas turbine and combined-cycle gas turbine units, due to reduced wear on the working surfaces of piston and screw compressors, booster compressor stations; prevent the products of internal corrosion of gas pipelines in gas-turbine units, in accordance with clause 93, 94 Federal standards and rules in the field of industrial safety "Safety Rules of gas distribution networks and gas consumption» (Series 12, vol. 13).

Object: a cyclone model based on CN-11-200, providing increased energy and environmental efficiency of gas treatment systems on thermal power plant.

Methods: bench tests of a cyclone filter manufactured by modernizing the cyclone CN-11-200, aerodynamic calculations; numerical simulation of aerodynamic parameters of the cyclone based on the methods of computational fluid dynamics. For mathematical modeling of separation the authors have used the system of equations consisting of the Reynolds-averaged Navier-Stokes equations of motion for single-phase flow and equations of particles motion based on Newton's law. To determine the effectiveness of separating the solid part of the flow in the cyclone-filter the dimensionless complex Re $e_{r}$ was used. Using the complex the numerical values of parameters from deposition of suspended solids from multiphase flow in the separator were determined by calculation.

The results shown lack of designs which would provide high (more than $98 \%$ ) extent of cleaning of emissions without sharp increase in energy consumption at processing emissions. The experiments carried out demonstrate the expediency of constructive addition of returnable and line cyclones with the filtering insert in a zone where there is an inertial sedimentation of dust. Extents of particle sedimentation in a cyclone depending on Reynolds number are defined. The calculations shown the compliance of results of theoretical and natural researches.
\end{abstract}

Key words:

Cyclone, filter, separation, extent of cleaning, modeling method, energy consumption, greenhouse gases.

\section{REFERENCES}

1. Krupneyshie mestorozhdeniya gaza $v$ Rossii [The largest gas deposits in Russia]. Available at: http://vivareit.ru/krupnejshie-mestorozhdeniya-gaza-v-rossii (accessed 04 April 2019).

2. Khamidullin R.N. Tekhnologiya ochistki gazovykh vybrosov ot pyli proizvodstva silikatnogo kirpicha. Avtoreferat Dis. Kand. nauk [Technology of cleaning of gas emissions of dust of production of a silicate brick. Cand. Diss. Abstract]. Kazan, 2005. 20 p.

3. Brunnmair E. Entwicklung und Modellierung eines neuen Hochleistungszyklons zur Trennung von Feststoff/Gas-Gemischen. Dokt. Diss. Leoben, 2015. 168 p.

4. Bielefeldt E.A. Wirbelkammern neurer Technologie [Eddy chambers of new technology]. Journal of Professional Issues in Engineering Education and Practice, 2013, vol. 15, pp. 286-291.

5. Muschelknautz E. Theorie der Fliehkraftabscheider mit besonderer Berücksichtigung hoher Temperaturen und Drücke [Theory of centrifugal separators with special consideration of high temperatures and pressures]. VDI-Bericht Nr., 1980, no. 383, pp. $49-60$.
6. Staudinger G. Zyklone mit Zentralkörpern Veröffentlichung [Cyclones with Central body publication]. Chem. Ing.Tech. Journal, 1992, vol. 64, pp. 769-888.

7. Ziganshin M.G. Tsiklon-filtr [Cyclone filter]. Patent RF, no. $2361678,2009$.

8. Balestrin E., Decker R.K., Noriler D., Bastos J.C.S.C., Meier H.F. An alternative for the collection of small particles in cyclones: experimental analysis and CFD modeling. Separation and Purification Technology, 2017, no. 5, pp. 54-65.

9. Mazyan W.I., Ahmadi A., Ahmed H., Hoorfar M. Increasing Efficiency of Natural Gas Cyclones through Addition of Tangential Chambers. Journal of Aerosol Science, 2017, vol. 4, no. 11, pp. 36-42.

10. Temnikova E.Yu., Bogomolov A.R., Petrik P.T. Issledovanie kharakteristik tsiklona s vnutrennimi elementami [Research of characteristics of a cyclone with internal elements]. Vestnik Kuzbasskogo gosudarstvennogo tekhicheskogo Universiteta, 2009, no. 2, pp. 140-144.

11. Serebryansky D.A., Zakharov A.A., Plashikhin S.V. Tsiklonnye pyleuloviteli. Malozatratnaya modernizatsiya [Cyclonic dust col- 
lectors. Low-cost modernization]. Chemical industry of Ukraine, 2013, vol. 3, pp. 70-74.

12. Atlas promyshlennykh pyley. Ch. 1. Letuchaya zola promyshlennykh elektrostantsiy [Atlas of industrial dusts. P. 1. Fly ash from industrial power plants]. Ed. by L.Ya. Scryabin. Moscow, TsINTIKhimneftemash Publ., 1980. 48 p.

13. Ziganshin M.G., Ziganshin A.M. Laboratorno-prakticheskiy kurs pylegazoochistki. Ch.1 [Laboratory and practical course of dust and gas cleaning]. Kazan, KGASU Publ. house, 2012.188 p.

14. Ziganshin M.G., Ziganshin A.M. Poluempiricheskaya model obrazovaniya avtosloya na tkannykh filtratsionnykh materialakh. Ch. 2. Opytnoe issledovanie filtratsii [Semiempirical model of education autolayaer on woven filtration materials. P. 2. Experimental study of filtration]. Izvestiya Kazanskogo gosudarstvennogo arkhitekturno-stroitelnogo universiteta, 2009, vol. 1, no. 11, pp. 186-190.

15. Eremkin A.I., Ziganshin M.G. Stepen inertsionnogo osazhdeniya vzveshennykh chastits v tsiklonnykh i vikhrevykh separatorakh. Verifikatsiya parametrov effektivnosti [The degree of inertia deposition of suspended particles in cyclone and vortex separators. Verification of performance parameters]. Nauchny vestnik $V_{0}$ ronezhskogo gosudarstvennogo arkhitekturno-stroitelnogo universiteta, 2009, vol. 3, no. 15, pp. 137-145.

16. Ziganshin M.G. Teoreticheskie osnovy pylegazoochistki [Theoretical bases of dusting]. Kazan, KGASU Publ. house, 2005. 262 p.

17. Ziganshin M.G., Kolesnik A.A., Ziganshin A.M. Proektirovanie apparatov pylegazoochistki [Design of dusting devices]. St. Petersburg, Lan Publ., 2014. 544 p.

18. Fassani F.L., Goldstein L. A study of the effect of high inlet solids loading on a cyclone separator pressure drop and collection efficiency. Powder Technology, 2000, vol. 3, no. 18, pp. 60-65.

19. Hoffmann C., Vansanten A., Allen R.W.K., Clift R. Effects of geometry and solid loading on the performance of gas cyclones. Powder Technology, 1992, no. 13, pp. 83-91.

20. Chu K.W., Wang B., Yu A.B., Vince A. CFD-DEM modeling of multiphase flow in dense medium cyclones. Powder Technology, 2009, vol. 1, no. 7, pp. 235-247.
21. Hamdy 0., Bassily M.A., El-Batsh H.M., Mekhail T.A. Numerical Study of the Effect of Changing the Cyclone Cone Length on the Gas Flow Field. Applied Mathematical Modelling, 2017, vol. 1, no. 26, pp. 81-97.

22. Wang B., Chu K.W., Yu A.B., Vince A. Modeling the multiphase flow in a dense medium cyclone. Industrial \& Engineering Chemistry Research, 2009, no. 48, pp. 3628-3639.

23. Zhou Z.Y., Kuang S.B., Chu K.W., Yu A.B. Assessments of CFDDEM models in particle fluid flow modeling. Journal of Fluid Mechanics, 2010, no. 661, pp. 482-510.

24. Ziganshin A.M., Gimadiyeva G.A., Batrova K.E. Chislennoe issledovanie istecheniya iz bokovogo poslednego i srednego otverstiy [Numerical research of expiration from side last and average openings]. Sbornik statey I Vserossijskoy nauchno-tekhnicheskoy konferentsii, posvyashchennoy pamyati doktora tekhnicheskikh nauk, professora A.A. Sandera [Collection of articles of the AllRussian scientific and technical conference devoted to memory of the Dr. Sc., professor A.A. Sander]. Novosibirsk, Novosibirsk state University of architecture and civil engineering, 2017. pp. 103-111.

25. Ziganshin A.M. Snizhenie energozatrat pri dvizhenii potokov putem profilirovaniya fasonnykh chastey v kommunikatsiyakh energoustanovok [Reduction of energy consumption in traffic flow by profiling fittings in communications power plants]. $\mathrm{Na}$ dezhnost i bezopasnost energetiki, 2015, vol. 1, no. 28, pp. 63-68.

26. Ziganshin A.M., Alechenko I.S. Soedinitelny fasonny element s profiliruyushchimi vstavkami [Connecting shaped element with profiling inserts]. Patent RF, no. 2604264, 2016.

27. Ziganshin A.M., Gimadieva A.G., Batova K.E. Poteri davleniya i kharakteristiki strui, vykhodyashchey cherez srednee bokovoe otverstie [Pressure drop and characteristics of the jet exiting through the secondary side opening]. Izvestiya KazGASU, 2017, vol. 4 , no. 42, pp. 257-265.

28. Logachev K.I., Ziganshin A.M., Averkova O.A., Logachev A.K. A survey of separated airflow patterns at inlet of circular exhaust hoods. Energy \& Buildings, 2018, no. 173, pp. 58-70.

Received: 27 August 2019.

\section{Information about the authors}

Albina T. Zamalieva, engineer, LLC «Gazprom transgaz Kazan».

Malik G. Ziganshin, Dr. Sc., associate professor, Kazan State Energy University. 